\title{
Efficacy of conversion therapy on initially unresectable locally advanced rectal cancer
}

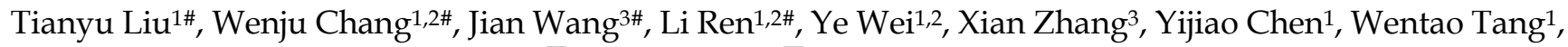
Mingliang Wang ${ }^{4}$, Zhaochong Zeng ${ }^{3}$, Jianmin $\mathrm{Xu}^{1,2}$

1. Colorectal Cancer Center, Department of General Surgery, Zhongshan Hospital, Fudan University, Shanghai, China.

2. Shanghai Engineering Research Center of Colorectal Cancer Minimally Invasive Technology (17DZ2252600), Shanghai, China.

3. Department of Radiotherapy; Zhongshan Hospital, Fudan University, Shanghai, China.

4. Department of Radiology, Zhongshan Hospital, Fudan University, Shanghai, China.

\# These authors contributed equally.

$\triangle$ Corresponding authors: Jianmin Xu (Email address: xujmin@aliyun.com), Department of General Surgery, Zhongshan Hospital, Fudan University; Zhaochong Zeng (Email address: zeng.zhaochong@zs-hospital.sh.cn), Department of Radiology, Zhongshan Hospital, Fudan University; Tel: +86-021-64061990; Fax: +86-021-64061990.

(C) The author(s). This is an open access article distributed under the terms of the Creative Commons Attribution License (https://creativecommons.org/licenses/by/4.0/). See http://ivyspring.com/terms for full terms and conditions.

Received: 2020.09.27; Accepted: 2021.05.11; Published: 2021.05.27

\begin{abstract}
Background and purpose: Research on the efficacy of conversion therapy for initially unresectable mid-low rectal cancer (IURC) remained limited. This study aimed to assess the efficacy and safety of the conversion regimen for IURC and analyze the long-term outcomes of these patients.

Methods: We retrospectively analyzed the data of clinically diagnosed IURC patients who received conversion therapy between October 2010 and April 2017. The conversion therapy consisted of long-term radiation, concurrent chemotherapy, delayed surgery and consolidation chemotherapy. The primary end point was the rate of R0 resection, and other short- and long-term outcomes were analyzed.

Results: Sixty-one patients were enrolled in this study. After conversion therapy, 51 (83.6\%) patients received R0 resection. The rates of pathologic complete response and downstaging were $16.4 \%$ and $62.3 \%$, respectively. The rate of grade $3-4$ chemoradiotherapy-related toxicity events was $13.1 \%$. The overall survival at 3 years was $75.4 \%$ in all patients, and the disease-free survival at 3 years was $72.5 \%$ in patients who received $\mathrm{R} 0$ resection.

Conclusion: The conversion regimen showed a high conversion resection rate and good survival outcomes in IURC patients, and might benefit the patients if recommended in clinical practice.
\end{abstract}

Key words: Unresectable; Rectal Cancer; Conversion Therapy; Prognosis.

\section{Introduction}

Neoadjuvant chemoradiotherapy and total mesorectal excision (TME) had dramatically reduced local recurrences and improved survival for patients with locally advanced mid-low rectal cancer [1, 2]. Most studies in this field only focused on resectable rectal cancer [3-5], leaving the niche - the efficacy in unresectable rectal cancer - to be filled. The treatment for initially unresectable mid-low rectal cancer (IURC), which is commonly defined as a palpably fixed lesion involving adjacent structures or as a large nonmobile tumor $[6,7]$, is to achieve tumor shrinkage and $\mathrm{R} 0$ resection, i.e., conversion therapy. However, with limited data, optimal strategy and the efficacy of the conversion therapy for IURC patients remained undetermined.

Several studies have claimed that some chemoradiotherapy strategies have potential to improve the rate of pathologic complete response (pCR) and local control of mid-low rectal cancer. For examples, studies revealed that addition of fluorouracil and leucovorin to preoperative radiotherapy improved local control, the rate of $\mathrm{R} 0$ resection and cancer-specific survival compared with preoperative radiotherapy alone [5, 7]. Also, 
CAO/ARO/AIO-94 study and FOWARC study indicated that adding oxaliplatin to neoadjuvant chemoradiotherapy significantly improved diseasefree survival (DFS) and pCR rate with acceptable acute toxicity $[8,9]$. Besides, delayed surgery or several cycles of mFOLFOX6 between the end of chemoradiotherapy and TME could give rise to tumor downstaging [10], increase the rates of $\mathrm{pCR}$ and $\mathrm{R} 0$ resection and improve DFS [10-13].

Based on the previous studies, we have developed a conversion regimen for patients with IURC, which is concurrent administration of oxaliplatin and oral fluoropyrimidines during conventionally fractionated radiotherapy, followed by several cycles of consolidation chemotherapy and delayed surgery. In this study, we retrospectively assessed the efficacy and safety of this regimen in IURC patients and analyzed the prognosis, providing new insights into the management of IURC patients.

\section{Materials and Methods}

\section{Patients}

Medical records of patients with mid-low rectal cancer admitted to Zhongshan Hospital Fudan University from October 2010 to April 2017 have been reviewed. Patients with initially unresectable mid-low rectal cancer, which was defined as a primary tumor involving adjacent organs or structures (cT4bNxM0) or palpably fixed cT3N2 tumor that could not be radically resected (fixed cT3N2M0), were analyzed. Written informed consent was obtained from all patients before the start of the study and this study was approved by the Institutional Ethics Committee of Zhongshan Hospital Fudan University, and was conducted in accordance with the Declaration of Helsinki.

The inclusion criteria included histologically confirmed adenocarcinoma of the rectum, distance $\leq 10$ centimeters from the anal verge to the inferior margin of the tumor determined by flexible colonoscopy, cT4b or fixed cT3N2 tumor without distant metastasis, and ASA score of 1-3. The exclusion criteria included history of previous malignant tumor, history of previous pelvic surgery, multiple colorectal cancers, severe diseases of other systems, and pregnant or breast-feeding women. The tumor height was measured by flexible colonoscopy, and the clinical stages of all patients were assessed by pelvic magnetic resonance imaging (MRI) and/or abdominopelvic computed tomography (CT).

\section{Conversion therapy regimen}

All patients were evaluated by the multidisciplinary team (MDT) before receiving any therapy and were recommended to receive the conversion therapy containing three parts: 1) radiotherapy: a total of $45 \mathrm{~Gy}$ in 25 fractions (1.8 Gy per day, 5 days a week for 5 weeks), followed by a minimum boost of 5.4 Gy; 2) concurrent chemotherapy: oral capecitabine, $825 \mathrm{mg} / \mathrm{m}^{2}$ twice a day from day 1 to 5 per week, and oxaliplatin, 50 $\mathrm{mg} / \mathrm{m}^{2}$ once per week at day $1,8,15,22,29$ by continuous infusion throughout radiation; 3 ) delayed surgery and the consolidation chemotherapy: patients received 3 cycles of mFOLFOX6 or 2 cycles of Capox one week after the completion of radiotherapy. Each cycle of mFOLFOX6 consisted of leucovorin 400 $\mathrm{mg} / \mathrm{m}^{2}$, oxaliplatin $85 \mathrm{mg} / \mathrm{m}^{2}$ in a 4-hour infusion, bolus fluorouracil $400 \mathrm{mg} / \mathrm{m}^{2}$ on day 1 , and a 48 -hour infusion of fluorouracil $2400 \mathrm{mg} / \mathrm{m}^{2}$. Each cycle of Capox consisted of capecitabine $\left(1000 \mathrm{mg} / \mathrm{m}^{2}\right.$ twice daily for 14 days every 3 weeks) and oxaliplatin (130 $\mathrm{mg} / \mathrm{m}^{2}$ in a 4 -hour infusion on day 1 of each cycle).

\section{Surgery and Pathologic Analysis}

The second resectability evaluation by the MDT was performed approximately 8 weeks after completion of the chemoradiotherapy. Operation was recommended to the patients whose tumor shrank to be resectable and TME was performed approximately 2 weeks after the last cycle of consolidation chemotherapy. For those patients whose tumors were progressive or still remained unresected, further treatment was given according to the advises of the MDT. The type of surgery was determined according to the situation of intraoperative exploration on the premise of radical resection. Multiple organ resection was performed when the tumor was invasive to adjacent organs. Preventive ileostomy was performed in patients with high anastomotic tension.

Pathologic stages were recorded according to AJCC Cancer Staging Manual, 8th edition and distal margin involvement was documented. Pathological complete response was defined as ypT0N0M0, and pathologic downstaging was defined as lower pathologic $\mathrm{T}$ stage compared with the pretreatment clinical $\mathrm{T}$ stage. Tumor response to the conversion therapy was documented according the Modified Ryan classification recommended by NCCN Guidelines.

\section{Follow-up}

All patients were routinely followed up every 3 to 6 months within the first two years, every half a year within 5 years and every year after 5 years. The examinations included serum carcinoembryonic antigen and abdominal ultrasonography at each time, CT scans every 6 to 12 months, and colonoscopy one year after operation and every 3 to 5 years. Local recurrence, distant metastasis and death event were 
documented once clinical or pathologic evidences were found.

\section{Statistics}

Overall survival (OS) was calculated from the initiation of conversion therapy to death of any cause or the latest follow-up. Disease-free survival (DFS) was calculated from the date of $\mathrm{R} 0$ resection to recurrence, metastasis or the latest follow-up. Survival curves were estimated by the Kaplan-Meier method and compared using the log-rank test. $P<$ 0.05 was considered to be statistically significant. All statistical evaluations were performed with SPSS software version 22.0.

\section{Results}

\section{Patients}

Sixty-one patients were included in the study. Patient baseline characteristics were showed in Table 1. The majority of the patients enrolled $(77.0 \%)$ had cT4b primary tumors. All the cT3N2 tumors were palpably fixed and had presacral space lymph nodes involvement, and all the cT4b tumors had at least one adjacent organ involvement identified by at least one experienced radiologist, which made them unresectable (Table 1).

Table 1. Baseline characteristics $(\mathrm{N}=61)$.

\begin{tabular}{ll}
\hline Characteristic & $\begin{array}{l}\text { Number of patients } \\
\text { n (\%) }\end{array}$ \\
\hline Sex & $40(65.6)$ \\
Male & $21(34.4)$ \\
Female & \\
Age (years) & $59.6(13.4)$ \\
Mean (SD) & \\
ASA score & $42(68.9)$ \\
I & $19(31.1)$ \\
II & $0(0)$ \\
III & \\
Distance between tumor and anal verge (cm) & $29(47.5)$ \\
$\leq 5$ & $32(52.5)$ \\
$>5$ and $\leq 10$ & \\
Clinical stages & $47(77.0)$ \\
cT4bNanyM0 * & $17(27.9)$ \\
Sacrum & $10(16.4)$ \\
Prostate gland & $8(13.1)$ \\
Uterus & $7(11.5)$ \\
Seminal vesicle gland & $7(11.5)$ \\
Base of the bladder & $6(9.8)$ \\
Pelvic side wall/floor & $1(1.6)$ \\
Posterior wall of vagina & $14(23.0)$ \\
cT3N2M0 & $14(23.0)$ \\
Lymph nodes in presacral space & \\
\hline Abbreviations: SD: standard deviation; ASA: American Society of \\
Anesthesiologists; cm: centimeter. * Details of the involvement of adjacent organs \\
or structures. Some tumors involving two or more organs or structures.
\end{tabular}

\section{Conversion therapy and toxicity events}

The regimen and completion status of conversion therapy were presented in Table 2. In summary, 1) The established full dose of radiation was performed in all of the 61 patients; 2 ) $62.3 \%$ of the patients received concurrent chemotherapy with capecitabine plus oxaliplatin and $37.7 \%$ with capecitabine alone; 3) 46 (75.4\%) patients received consolidation chemotherapy with mFOLFOX6 or Capox after the end of radiation, and $6(9.8 \%)$ received capecitabine alone. The median number of cycles of consolidation chemotherapy was 2 (range from 1 to 3 ).

Acute adverse effects of the conversion therapy were showed in Table 3. Overall toxicity (grade 1-4) was observed in $29(47.5 \%)$ patients, including that grade 1-2 toxicity occurred in $21(34.4 \%)$ patients and grade 3 toxicity occurred in $8(13.1 \%)$ patients. No grade 4 complication or treatment-related death has occurred. Bone marrow depression was the most common toxic effect occurred during the chemoradiotherapy, leading to drug withdrawal or drug reduces. Diarrhea was observed in 8 patients, and 2 of them reduced the dose of capecitabine.

Table 2. Regimen of conversion therapy $(\mathrm{N}=61)$.

\begin{tabular}{ll}
\hline Characteristics & $\begin{array}{l}\text { Number of patients } \\
\mathbf{n}(\%)\end{array}$ \\
\hline $\begin{array}{l}\text { Radiotherapy } \\
\text { Concurrent chemotherapy regimen }\end{array}$ & $61(100)$ \\
Capecitabine and oxaliplatin & $38(62.3)$ \\
Capecitabine alone & $23(37.7)$ \\
Concurrent chemotherapy with dose reduction* & $7(11.5)$ \\
Consolidation chemotherapy regimen & \\
mFOLFOX6 & $40(65.6)$ \\
Capox & $6(9.8)$ \\
Capecitabine & $6(9.8)$ \\
None & $9(14.8)$ \\
Cycles of consolidation chemotherapy & \\
0 & $9(14.8)$ \\
1 & $15(24.6)$ \\
2 & $14(23.0)$ \\
3 & $23(37.7)$
\end{tabular}

Table 3. Complications related to conversion therapy $(\mathrm{N}=61)$.

\begin{tabular}{|c|c|c|c|}
\hline Events * & $\begin{array}{l}\text { All } \\
\text { n (\%) }\end{array}$ & $\begin{array}{l}\text { Grade 1-2 } \\
\text { n (\%) }\end{array}$ & $\begin{array}{l}\text { Grade3 } \\
\text { n (\%) }\end{array}$ \\
\hline Toxicity (NCI-CTC version 3.0) & $29(47.5)$ & $21(34.4)$ & $8(13.1)$ \\
\hline \multicolumn{4}{|l|}{ Hematological } \\
\hline Leucopenia & $10(16.4)$ & $8(13.1)$ & $2(3.3)$ \\
\hline Anemia & $3(4.9)$ & $1(1.6)$ & $2(3.3)$ \\
\hline Infection or fever & $3(4.9)$ & $2(3.3)$ & $1(1.6)$ \\
\hline \multicolumn{4}{|l|}{ Gastrointestinal } \\
\hline Diarrhea & $8(13.1)$ & $5(8.2)$ & $3(4.9)$ \\
\hline Nausea or vomiting & $2(3.3)$ & $1(1.6)$ & $1(1.6)$ \\
\hline Radiation procitis & $9(14.8)$ & $8(13.1)$ & $1(1.6)$ \\
\hline Hand-foot syndrome & $4(6.6)$ & $3(4.9)$ & $1(1.6)$ \\
\hline Radiation dermatitis & $8(13.1)$ & $7(11.5)$ & $1(1.6)$ \\
\hline
\end{tabular}

\section{Response and surgery}

At the second resectability evaluation, 54 (88.5\%) patients had tumor size reduction, while $3(4.9 \%)$ patients had stable-disease response, $3(4.9 \%)$ had 
local progression and 1 (1.6\%) had osseous metastasis. All of the 54 patients received surgery, and 51 (83.6\%) of them received $\mathrm{R} 0$ resection, while 3 of them still had unresectable lesions determined during the operation and received alternative surgery, including 2 patients underwent sigmoidostomy and one received $\mathrm{R} 2$ resection. The median interval between completion of chemoradiotherapy and surgery was 64.5 days (IQR 57.5-72.5). After conversion therapy, a high response rate was observed in terms of the tumor regression grade (TRG) 0 to 1 . Pathological complete response and downstaging were found in $10(16.4 \%)$ and $38(62.3 \%)$ patients, respectively. Details were shown in Table 4.

Table 4. Response of conversion therapy $(\mathrm{N}=61)$.

\begin{tabular}{ll}
\hline Characteristics & $\begin{array}{l}\text { Number of patients } \\
\mathbf{n}(\%)\end{array}$ \\
\hline Clinical evaluation & \\
Clinical downstaging & $54(88.5)$ \\
Stable disease & $3(4.9)$ \\
Local progression & $3(4.9)$ \\
Distant metastases & $1(1.6)$ \\
Received surgery & \\
No & $7(11.5)$ \\
Yes & $54(88.5)$ \\
$<8 \mathrm{w}$ & $9(14.8)$ \\
$\geq 8 \mathrm{w} ;<12 \mathrm{w}$ & $37(60.7)$ \\
$\geq 12 \mathrm{w}$ & $8(13.1)$ \\
Conversion results \# & \\
Successful & $51(83.6)$ \\
Fail & $10(16.4)$ \\
No surgery & $7(11.5)$ \\
R2 resection & $1(1.6)$ \\
Sigmoidostomy & $2(3.3)$ \\
Tumor regression grade & \\
0 & $10(16.4)$ \\
1 & $21(34.4)$ \\
2 & $5(8.2)$ \\
3 & $15(24.6)$ \\
pCR & $10(16.4)$ \\
Pathological downstaging & $38(62.3)$ \\
\hline Abbreviations: RT: radiotherapy; w: week; pCR: pathological complete response. \\
*Interval between the completion of chemoradiotherapy and TME for patients who \\
received surgery \\
\# Successful conversion was defined as R0 resection after conversion therapy. Fail \\
conversion was defined as still unresectable tumor after conversion therapy. \\
(n)
\end{tabular}

A Overall survival

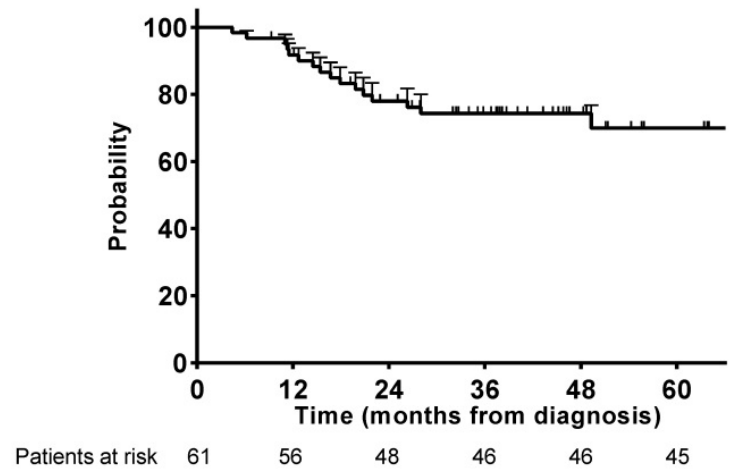

\section{Short-term outcomes and surgical complications}

Operative details of patients who underwent R0 resection were shown in Table 5. Minimally invasive surgery was performed in $32(62.7 \%)$ patients and the incidence of sphincter-sparing resection was $49 \%$. The rate of combined organ resection, the operation time and the intraoperative blood loss were acceptable. Preventive ileostomy was performed in 3 patients. The postoperative acute adverse events were showed in Supplementary Table 1. These data suggested that the short-term outcomes were acceptable in the successful conversion patients.

Table 5. Short-term outcomes for the successful conversion patients $(\mathrm{N}=51)$.

\begin{tabular}{ll}
\hline Characteristics & $\begin{array}{l}\text { Successful conversion } \\
\mathbf{n}(\%)\end{array}$ \\
\hline Surgical procedures & \\
Abdominoperineal resection & $26(51.0)$ \\
Low anterior resection & $23(45.1)$ \\
Hartmann & $2(3.9)$ \\
Minimally invasive surgery & \\
Yes & $32(62.7)$ \\
No & $19(37.3)$ \\
Protective ileostomy & $3 / 23(13.0)$ \\
Combined organ resection & $4(7.8)$ \\
Operative time (min) & $162.5 \pm 22.7$ \\
Intraoperative blood loss (ml) & $74 \pm 59$ \\
Postoperative complications & $18(35.2)$ \\
Reoperations & $0(0)$ \\
Hospitalized days after surgery, median (range) & $7(4-28)$ \\
Urinary catheter, (day) & $4.1 \pm 3.9$ \\
Liquid diet, (day) & $3.3 \pm 2.5$ \\
\hline
\end{tabular}

\section{Long-term survival}

The median follow-up time was 37.6 months overall. Seventeen patients died during follow-up and the OS rate at 3 years was $75.4 \%$ (Figure $1 \mathrm{~A}$ ). Significant survival benefit was observed in the successful conversion patients compared with those who failed to the conversion therapy (Figure 1B).

B

Overall survival

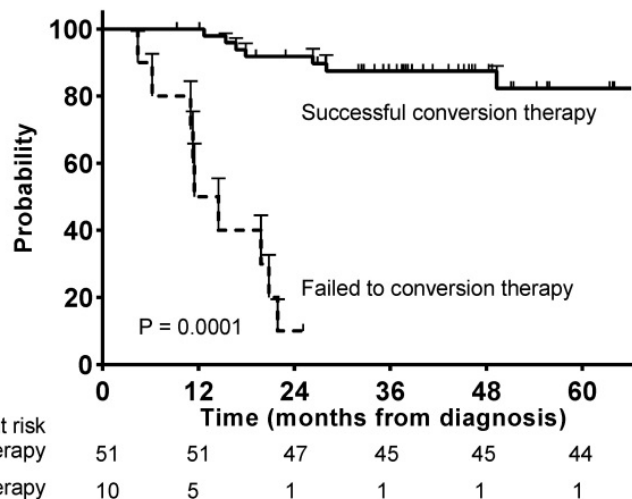

Figure 1. Overall survivals. (A) Overall survival for the patients who received conversion therapy; (B) Overall survival for the successful conversion patients and failed conversion patients. 


\section{Disease free survival}

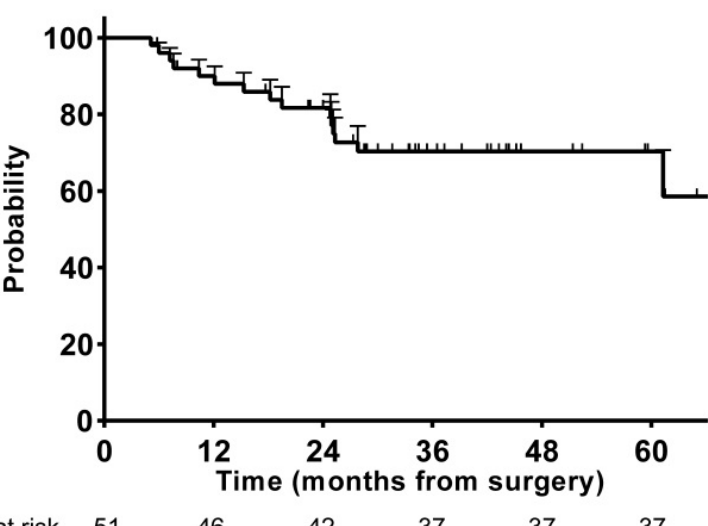

$\begin{array}{lllllll}\text { Patients at risk } & 51 & 46 & 42 & 37 & 37 & 37\end{array}$

Figure 2. Disease-free survival for the successful conversion patients.

For patients who received R0 resection, total recurrence events occurred in $15(29.4 \%)$ of the 51 patients. The DFS rate at 3 years was $72.5 \%$ in the successful conversion patients (Figure 2).

\section{Discussion}

To our knowledge, the standard regimen for IURC patients and its outcomes still remained unclear. This study showed that patients with initially unresectable mid-low rectal cancer treated with this conversion therapy achieved a high rate of $\mathrm{R} 0$ resection with an desirable long-term survival and a low rate of grade 3-4 toxicity. This study could provide some reference for optimizing the management of IURC patients.

The rate of $\mathrm{R} 0$ resection in this study was higher than those in previous studies with similar population characteristics. Bujko and colleagues reported the rates to be $71 \%-77 \%$ in their study with two groups of conversion therapy [14], which specifically were $77 \%$ in the group treated with $5 \times 5$ Gy and three cycles of FOLFOX 4 and $71 \%$ in the group treated with long-course simultaneously chemoradiotherapy. The better performance of our regimen could be attributed to the following strategies adopted in it. First, the delayed surgery was used in our regimen, which has been reported to be able to induce tumor downstaging $[10-12,15,16]$. Second, our patients received consolidation chemotherapy during the interval of chemoradiotherapy and TME, which had been confirmed efficient in improving the $\mathrm{pCR}$ rate in previous studies [13, 17]. Third, comparing to the former group which used short-course radiotherapy with 3 cycles of FOLFOX4, our R0 resection rate was slightly higher, probably because the concurrent chemotherapy may enhance the efficacy of preoperative treatment [18], even though no direct evidence was found to demonstrate the superiority of conventionally fractionated radiation to short-course radiation $[16,19]$. Finally, the addition of oxaliplatin to fluorouracil-based chemotherapy has been reported to be more efficient in preoperative treatment $[8,9]$.

Upon reviewing previous literatures for optimization of therapeutic strategies for nonresectable rectal cancer, we found that the successful conversion rate $(83.6 \%)$ in our study was the same as that $(83.6 \%)$ reported by a previous randomized controlled clinical trial [7], though our strategy was more aggressive. However, the inclusion criteria differed between the two studies, and the cases we were dealing with might be more challenging. In the previous studies, patients with fixed cT3 or cT4 lesions and recurrence tumor were enrolled, while in this study, the eligible patients had cT4b tumor or fixed cT3 tumor with lymph node metastases (fixed cT3N2) confirmed by baseline MRI and/or CT. Obviously, by adding lymph node metastasis (N stage) into cT3 tumor enrollment, the inclusion criteria in this study was stricter.

Comparing to previous studies, the long-term outcomes of this conversion therapy were desirable. The 3-year OS rate in unresectable rectal cancer patients reported in previous studies was 53\% - 73\% $[7,14]$. For another, the 3-year DFS rate reported in the FOWARC trial and the German CAO/ARO/AIO-04 study was $71.2 \%-77.2 \%[8,20]$. In this study, the 3 -year OS rate and DFS rate were $75.4 \%$ and $72.5 \%$, respectively, suggesting competitive efficacy of this conversion regimen.

The toxicity of this conversion regimen was acceptable. For one thing, the rate of chemoradiotherapy related grade 3-4 acute toxic effects $(13.1 \%)$ was lower compared to the previously studies of preoperative chemoradiotherapy with oxaliplatin or consolidation chemotherapy (18\%-40\%) $[3,8,9,13,21-23]$. The lower rate of toxicity could be attributed to the application of image-guided intensity-modulated radiotherapy which entailed higher target conformity and lower radiographic exposure of bowel and bladder [24, 25]. For another, the postoperative complications occurred in 18 (35.2\%) patients who achieved R0 resection, which was similar to the STAR-01 trial and the NSAPB R-04 trial $[3,23]$.

There are some limitations in this study. First, it was a retrospective study with a limited sample size. Second, $16.4 \%$ of the patients failed to obtain initial pelvic MRI at baseline due to implanted metal objects in patient's body or other reasons, and alternatively, contrast CT was performed and the results were evaluated by two experienced radiologists. 


\section{Conclusion}

In conclusion, this study proposed a conversion regimen which was proved safe and effective for IURC patients, contributing to high rate of R0 resection and superior survival outcomes. Although lacking clinical evidences of higher levels, this regimen definitely has promising performance on IURC treatment and may improve survival benefits.

\section{Supplementary Material}

Supplementary table.

http://www.jcancer.org/v12p4418s1.pdf

\section{Acknowledgements}

This study was funded by The National Natural Science Foundation of China (81602035, 81472228, 82072678); Shanghai Science and Technology Committee Project (19511121301); The Shanghai Municipal Commission of Health and Family Planning: Shanghai Outstanding Youth Specialist Training Program (Q2017-059); Clinical Science and Technology Innovation Project of Shanghai (SHDC12016104); Clinical Research Plan of SHDC (SHDC2020CR5006, SHDC2020CR1033B, SHDC2020 CR3037B).

\section{Competing Interests}

There was no financial and personal relationship with other people and organizations that could inappropriately influence this work.

\section{References}

1. Heald RJ, Ryall RD. Recurrence and survival after total mesorectal excision for rectal cancer. Lancet. 1986; 1(8496): 1479-1482.

2. Sauer R, Becker H, Hohenberger W, Rodel C, Wittekind C, Fietkau R, et al. Preoperative versus postoperative chemoradiotherapy for rectal cancer. N Engl J Med. 2004; 351(17): 1731-1740.

3. Aschele C, Cionini L, Lonardi S, Pinto C, Cordio S, Rosati G, et al. Primary tumor response to preoperative chemoradiation with or without oxaliplatin in locally advanced rectal cancer: pathologic results of the STAR-01 randomized phase III trial. J Clin Oncol. 2011; 29(20): 2773-2780.

4. Bosset JF, Calais G, Mineur L, Maingon P, Stojanovic-Rundic S, Bensadoun RJ, et al. Fluorouracil-based adjuvant chemotherapy after preoperative chemoradiotherapy in rectal cancer: long-term results of the EORTC 22921 randomised study. Lancet Oncol. 2014; 15(2): 184-190.

5. Gerard JP, Conroy T, Bonnetain F, Bouche O, Chapet O, Closon-Dejardin MT, et al. Preoperative radiotherapy with or without concurrent fluorouracil and leucovorin in T3-4 rectal cancers: results of FFCD 9203. J Clin Oncol. 2006; 24(28): 4620-4625.

6. Bujko K, Nasierowska-Guttmejer A, Wyrwicz L, Malinowska M, Krynski J, Kosakowska E, et al. Neoadjuvant treatment for unresectable rectal cancer: an interim analysis of a multicentre randomized study. Radiother Oncol. 2013; 107(2): 171-177.

7. Braendengen M, Tveit KM, Berglund A, Birkemeyer E, Frykholm G, Pahlman $\mathrm{L}$, et al. Randomized phase III study comparing preoperative radiotherapy with chemoradiotherapy in nonresectable rectal cancer. J Clin Oncol. 2008; 26(22): 3687-3694.

8. Rodel C, Graeven U, Fietkau R, Hohenberger W, Hothorn T, Arnold D, et al. Oxaliplatin added to fluorouracil-based preoperative chemoradiotherapy and postoperative chemotherapy of locally advanced rectal cancer (the German $\mathrm{CAO} / \mathrm{ARO} / \mathrm{AIO}-04$ study): final results of the multicentre, open-label, randomised, phase 3 trial. Lancet Oncol. 2015; 16(8): 979-989.

9. Deng Y, Chi P, Lan P, Wang L, Chen W, Cui L, et al. Modified FOLFOX6 With or Without Radiation Versus Fluorouracil and Leucovorin With Radiation in Neoadjuvant Treatment of Locally Advanced Rectal Cancer: Initial Results of the Chinese FOWARC Multicenter, Open-Label, Randomized Three-Arm Phase III Trial. J Clin Oncol. 2016; 34(27): 3300-3307.

10. Pettersson D, Lorinc E, Holm T, Iversen H, Cedermark B, Glimelius B, et al. Tumour regression in the randomized Stockholm III Trial of radiotherapy regimens for rectal cancer. Br J Surg. 2015; 102(8): 972-978; discussion 8.

11. Tulchinsky H, Shmueli E, Figer A, Klausner JM, Rabau M. An interval >7 weeks between neoadjuvant therapy and surgery improves pathologic complete response and disease-free survival in patients with locally advanced rectal cancer. Ann Surg Oncol. 2008; 15(10): 2661-1667.

12. Macchia G, Gambacorta MA, Masciocchi C, Chiloiro G, Mantello G, di Benedetto $\mathrm{M}$, et al. Time to surgery and pathologic complete response after neoadjuvant chemoradiation in rectal cancer: A population study on 2094 patients. Clin Transl Radiat Oncol. 2017; 4: 8-14.

13. Garcia-Aguilar J, Chow OS, Smith DD, Marcet JE, Cataldo PA, Varma MG, et al. Effect of adding mFOLFOX6 after neoadjuvant chemoradiation in locally advanced rectal cancer: a multicentre, phase 2 trial. Lancet Oncol. 2015; 16(8): 957-966.

14. Bujko K, Wyrwicz L, Rutkowski A, Malinowska M, Pietrzak L, Krynski J, et al. Long-course oxaliplatin-based preoperative chemoradiation versus 5 x 5 Gy and consolidation chemotherapy for cT4 or fixed cT3 rectal cancer: results of a randomized phase III study. Ann Oncol. 2016; 27(5): 834-842.

15. Francois $Y$, Nemoz CJ, Baulieux J, Vignal J, Grandjean JP, Partensky C, et al. Influence of the interval between preoperative radiation therapy and surgery on downstaging and on the rate of sphincter-sparing surgery for rectal cancer: the Lyon R90-01 randomized trial. J Clin Oncol. 1999; 17(8): 2396.

16. Erlandsson J, Holm T, Pettersson D, Berglund A, Cedermark B, Radu C, et al. Optimal fractionation of preoperative radiotherapy and timing to surgery for rectal cancer (Stockholm III): a multicentre, randomised, non-blinded, phase 3, non-inferiority trial. Lancet Oncol. 2017; 18(3): 336-346.

17. Fokas E, Allgauer M, Polat B, Klautke G, Grabenbauer GG, Fietkau R, et al. Randomized Phase II Trial of Chemoradiotherapy Plus Induction or Consolidation Chemotherapy as Total Neoadjuvant Therapy for Locally Advanced Rectal Cancer: CAO/ARO/AIO-12. J Clin Oncol. 2019: Jco1900308.

18. Yu Y, Li Y, Xu C, Zhang Z, Zhang X. Comparison of long course and short course preoperative radiotherapy in the treatment of locally advanced rectal cancer: a systematic review and meta-analysis. Rev Esp Enferm Dig. 2019; 111(1): 17-27.

19. Ngan SY, Burmeister B, Fisher RJ, Solomon M, Goldstein D, Joseph D, et al. Randomized trial of short-course radiotherapy versus long-course chemoradiation comparing rates of local recurrence in patients with T3 rectal cancer: Trans-Tasman Radiation Oncology Group trial 01.04. J Clin Oncol. 2012; 30(31): 3827-3833

20. Yanhong D, Pan C, Ping L, et al. Neoadjuvant Modified FOLFOX6 With or Without Radiation Versus Fluorouracil Plus Radiation for Locally Advanced Rectal Cancer: Final Results of the Chinese FOWARC Trial. J Clin Oncol. 2019; 37(34): 3223-3233.

21. Gerard JP, Azria D, Gourgou-Bourgade S, Martel-Laffay I, Hennequin C, Etienne PL, et al. Comparison of two neoadjuvant chemoradiotherapy regimens for locally advanced rectal cancer: results of the phase III trial ACCORD 12/0405-Prodige 2. J Clin Oncol. 2010; 28(10): 1638-1644.

22. Rodel C, Liersch T, Becker H, Fietkau R, Hohenberger W, Hothorn T, et al. Preoperative chemoradiotherapy and postoperative chemotherapy with fluorouracil and oxaliplatin versus fluorouracil alone in locally advanced rectal cancer: initial results of the German CAO/ARO/AIO-04 randomised phase 3 trial. Lancet Oncol. 2012; 13(7): 679-687.

23. O'Connell MJ, Colangelo LH, Beart RW, Petrelli NJ, Allegra CJ, Sharif S, et al. Capecitabine and oxaliplatin in the preoperative multimodality treatment of rectal cancer: surgical end points from National Surgical Adjuvant Breast and Bowel Project trial R-04. J Clin Oncol. 2014; 32(18):1927-1934.

24. Guerrero Urbano MT, Henrys AJ, Adams EJ, Norman AR, Bedford JL, Harrington $\mathrm{KJ}$, et al. Intensity-modulated radiotherapy in patients with locally advanced rectal cancer reduces volume of bowel treated to high dose levels. Int J Radiat Oncol Biol Phys. 2006; 65(3): 907-916.

25. Huang CM, Huang MY, Tsai HL, Huang CW, Ma CJ, Lin $\mathrm{CH}$, et al. A retrospective comparison of outcome and toxicity of preoperative image-guided intensity-modulated radiotherapy versus conventional pelvic radiotherapy for locally advanced rectal carcinoma. J Radiat Res. 2017; 58(2): 247-259. 